\title{
Enquête Ethnobotanique Des Plantes Utilisées Pour La Protection Cutanée Des Personnes Atteintes D'albinisme Dans Le District De Bamako (Mali) Et Analyse Qualitative De Bixa Orellana L. (Bixaceae)
}

\author{
Haidara Mahamane, \\ Amani Corina-Ardine, \\ Mariko Ä̈chata Ben Adam,
}

Faculté de Pharmacie, Université des Sciences des Techniques et des

Technologies de Bamako (USTTB), Bamako Mali

Gassama Mamadou,

Faculté de Médecine et Odontostomatologie, Université des Sciences des

Techniques et des Technologies de Bamako (USTTB), Bamako Mali

\section{Traore Mahamadou,}

Faculté de Pharmacie, Université des Sciences des Techniques et des

Technologies de Bamako (USTTB), Bamako Mali

\section{Sanogo Rokia,}

Faculté de Pharmacie, Université des Sciences des Techniques et des

Technologies de Bamako (USTTB), Bamako Mali

Département de Médecine Traditionnelle, Bamako Mali

Doi:10.19044/esj.2020.v16n12p370 URL:http://dx.doi.org/10.19044/esj.2020.v16n12p370

\section{Résumé}

Objectif : Cette étude avait pour objectif de recenser les plantes médicinales utilisées pour la protection cutanée des personnes atteintes d'albinisme, dans le district de Bamako (Mali). Matériel et méthodes : A l'aide d'un questionnaire, des informations ont été collectées auprès des membres d'a associations des personnes atteintes d'albinisme. Les plantes les plus citées ont été soumis à une revue bibliographique. Les constituants chimiques et antiradicalaires des graines de Bixa orellana ont été déterminés par les caractérisations en tubes et par la chromatographie sur couche mince. Résultats : Au total, Dix-neuf (19) plantes médicinales ont été recensées auprès de 75 personnes atteintes d'albinisme et sont principalement utilisées dans la protection cutanée. Les plantes les plus fréquemment citées ont été Khaya senegalensis (12 citations), Guiera senegalensis (10 citations), Bixa orellana (8 citations) et Vitellaria paradoxa (5 citations). Les caroténoïdes, les mucilages, les stérols, les triterpènes et les substances antiradicalaires ont 
été caractérisés dans les extraits des graines de Bixa orellana. Conclusion : Ces extraits peuvent servir à la formulation de produits qui vont contribuer à la photoprotection, l'hydratation et la protection cutanée des personnes atteintes d'albinisme.

Mots clés: Personne Atteinte d'Albinisme, Protection Cutanée, Bixa Orellana, Caroténoïdes, Mali

\title{
Ethnobotanical Survey of Plants used for Skin Protection of People with Albinism in the District of Bamako (Mali) and Qualitative Analysis of Bixa Orellana L. (Bixaceae)
}

\author{
Haidara Mahamane, \\ Amani Corina-Ardine, \\ Mariko Ä̈chata Ben Adam,
}

Faculté de Pharmacie, Université des Sciences des Techniques et des

Technologies de Bamako (USTTB), Bamako Mali

Gassama Mamadou,

Faculté de Médecine et Odontostomatologie, Université des Sciences des

Techniques et des Technologies de Bamako (USTTB), Bamako Mali

\section{Traore Mahamadou,}

Faculté de Pharmacie, Université des Sciences des Techniques et des

Technologies de Bamako (USTTB), Bamako Mali

\section{Sanogo Rokia,}

Faculté de Pharmacie, Université des Sciences des Techniques et des

Technologies de Bamako (USTTB), Bamako Mali

Département de Médecine Traditionnelle, Bamako Mali

\begin{abstract}
Objective: The objective of this study was to identify medicinal plants used for the skin protection of people with albinism, in the district of Bamako (Mali). Material and methods: Information was collected from members of associations of people with albinism using a questionnaire. The most cited plants were subjected to a bibliographic review. The chemical and anti-free radical components of Bixa orellana seeds were determined by color reactions and by thin layer chromatography. Results: In total, nineteen (19) medicinal
\end{abstract}


plants were identified among 75 people with albinism and are mainly used in skin protection. The most frequently mentioned plants were Khaya senegalensis (12 citations), Guiera senegalensis (10), Bixa orellana (8) and Vitellaria paradoxa (5). Carotenoids, mucilages, sterols, triterpenes and antifree radicals components have been characterized in extracts from the seeds of Bixa orellana. Conclusion: These extracts can be used to formulate products that will contribute to photoprotection, hydration and skin protection for people with albinism.

Keywords: Person With Albinism, Skin Protection, Bixa Orellana, Carotenoids, Mali

\section{Introduction}

L'albinisme regroupe un ensemble d'affections héréditaires liées à une anomalie de biosynthèse de la mélanine, pigment élaboré dans des cellules spécialisées de la peau, des cheveux, de l'iris, de l'épithélium pigmenté de la rétine et de l'oreille interne, associées à un nombre et à une structure normale des mélanocytes [Rooryck et al., 2007].

La réduction ce pigment est responsable d'une sensibilité accrue aux rayonnements UV et d'une prédisposition aux cancers de la peau.

L'albinisme oculo-cutané (AOC) comporte une hypopigmentation cutanéo-phanérienne généralisée et une atteinte ophtalmologique. Elle est la forme la plus fréquente d'albinisme [Rooryck et al., 2007].

La prévalence de toutes les formes d'albinismes varie considérablement dans le monde et a été estimé à environ à un cas sur 17000. Une personne sur 70 est porteuse d'un gène de type AOC [Grønskov et al., 2007]. Les estimations de l'Organisation mondiale de la Santé (OMS) oscillent entre un cas sur 5000 et un cas sur 15000 en Afrique subsaharienne [https://www.un.org/fr/]. Des études affirment que la plupart des personnes atteintes d'albinisme de type AOC meurent d'un cancer de la peau entre 30 et 40 ans [https://www.un.org/fr/events/albinismday/background.shtml].

C'est dans ce contexte que les personnes atteintes d'albinisme doivent bénéficier d'un suivi particulier pour leur protection, pour la prévention et la prise en charge des troubles ophtalmologique et dermatologique.

Pour améliorer la qualité de vie des personnes atteintes d'albinisme, un accent particulier est mis sur la protection contre les méfaits du soleil par le port de vêtements en coton, recouvrant les parties à fortes exposition solaire et par l'application de produits solaires à base d'agents photo-protecteurs.

Au Mali, la majorité de ces produits de protection sont importés et ne sont pas toujours accessibles aux personnes atteintes d'albinisme.

La présente étude a pour objectif d'étudier les plantes utilisées pour la protection cutanée des personnes atteintes d'albinisme dans le district de Bamako. 
Pour cela une enquête ethnobotanique a été menée afin de recenser les espèces végétales utilisées dans la protection et par la suite une des plantes les plus citée, a été sélectionnée pour des études phytochimiques.

\section{Méthodologie}

\section{Enquête}

Cette enquête a été menée auprès des personnes atteintes d'albinisme au sein des associations dans le district de Bamako et au niveau du Centre national d'appui à la maladie (CNAM) actuelle hôpital dermatologique du Mali.

\subsection{Structures enquêtées :}

$>$ Associations de lutte pour l'albinisme à Bamako (Mali): AMPA Mali, Fondation Salif Keita et SOS albinos Mali.

$>$ Le CNAM actuelle hôpital dermatologique du Mali

Ces structures ont été identifiées grâce une collaboration avec la présidente de l'AMPA Mali qui nous a donné la liste des associations de lutte pour l'albinisme à Bamako. Une lettre d'autorisation d'enquête a été ainsi adressée aux responsables de chaque structure avant de procédé à l'enquête, pour leurs expliquer les motivations de l'étude. Les structures ayant donné leur accord ont été retenues pour l'enquête.

\subsection{Collecte et analyse des données :}

Les données ont été collectées auprès des personnes atteintes d'albinisme oculo-cutané en utilisant un questionnaire semi -structuré. La fiche d'enquête comportait entre autres des questions sur le profil sociodémographique des participants, le recours ou pas à la phytothérapie, le nom local en Bamanan des plantes utilisées par les personnes atteintes d'albinisme oculo-cutané pour la protection et l'entretien de la peau. Les entretiens ont été réalisés par interrogation orale en français et ou en langue locale "Bamanan".

Les feuilles de calcul Excel ont été utilisées pour la saisie des données et pour effectuer des calculs simples et déterminer le sex ratio, le pourcentage (\%) d'ethnie et le pourcentage de recours à la phytothérapie.

$$
\begin{gathered}
\text { Sex ratio }=\frac{\text { Nombre de répondant homme }}{\text { Nombre de répondant femme }} \\
\% \mathrm{~d}^{\prime} \text { ethnie }=\frac{\text { Nombre de répondant pour une ethnie }}{\text { Nombre de total de repondant }} \times 100 \\
\% \text { de personne de recours à la phytothérapie }=\frac{\mathrm{n}}{\mathrm{N}} \times 100
\end{gathered}
$$


Où $\mathrm{n}=$ Nombre de répondant ayant eu recours à la phytothérapie et $\mathrm{N}=$ Nombre total de répondant

Le nom scientifique et leur famille botanique ont été déterminé en utilisant la base de donnée the Plant List [www.theplantlist.org].

\subsection{Considérations éthiques}

Avant de procédé à l'enquête, les motivations de l'étude ont été expliquées. Pour des considérations éthiques, les différents responsables des associations ont donné leur accord et le consentement verbal des personnes atteintes d'albinisme a été enregistré.

\section{Sélection de Bixa orellana L. (Bixaceae)}

A partir des données collectées, Bixa orellana a été sélectionnée pour deux raison :

$>$ Elle était l'une des plantes la plus fréquemment citée ;

$>$ Elle est riche en caroténoïdes selon la littérature [Zarza-García et al., 2017].

L'analyse phytochimique a été réalisée sur Bixa orellana au niveau du laboratoire du Département de Médecine Traditionnelle (DMT).

\section{Etude au laboratoire}

\subsection{Matériel végétal}

Le matériel végétal était constitué d'un échantillon de graines de Bixa orellana, acheté au marché de Médina coura Bamako. Ces graines ont été séchées pendant deux semaines dans la salle de séchage du DMT. Après séchage, ces graines ont été par la suite pilées au mortier. La poudre obtenue a servi pour les analyses physicochimiques quantitatives et pour la préparation des extraits.

\subsection{Analyse physicochimique quantitative de Bixa Orellana L. (Bixaceae)}

La teneur en eau a été déterminée par dessiccation à l'étuve à $103 \pm 2{ }^{\circ} \mathrm{C}$ pendant 24 heures. La teneur en cendres totales et cendres insolubles dans l'acide chlorhydrique $10 \%$ ont été déterminées par calcination au four à $600^{\circ} \mathrm{C}$ pendant 6 heures [N'Guessan Bra et al., 2015].

\subsection{Préparation des extraits}

\section{$>$ Infusion 10\%:}

Un total de $5 \mathrm{~g}$ de poudre des graines a été infusé dans $50 \mathrm{~mL}$ d'eau distillée bouillante pendant 15 minutes. Le mélange a été filtré en utilisant un papier filtre Whatman $\mathrm{N}^{\circ} 1$. 
$>$ Macération 10\%:

Un total de $5 \mathrm{~g}$ de poudre des graines a été macéré dans $50 \mathrm{~mL}$ d'éther de pétrole, ou d'éthanol $96 \%$ pendant 24 heures à la température ambiante. Le mélange a été filtré en utilisant un papier filtre Whatman $\mathrm{N}^{\circ} 1$.

Chaque filtrat a été concentré à l'évaporateur rotatif (Rotavapor), jusqu'à obtenir un volume d'environ $1 \mathrm{~mL}$ d'extrait qui a été conservé dans des flacons en verre avant leur utilisation pour la chromatographie sur couche mince.

\subsection{Analyse physicochimique qualitative de Bixa Orellana L. (Bixaceae)}

\subsubsection{Caractérisation des constituants chimiques}

La caractérisation des métabolites secondaires des graines de Bixa orellana a été fait par les réactions colorées en tube et par chromatographie sur couche mince (CCM).

\subsubsection{Réactions colorées en tube,}

Les réactifs de caractérisation classiques ont été utilisés pour mettre en évidence les groupes chimiques suivants : alcaloïdes (réactif de Dragendorff), anthracénosides (réaction de Bornträger), caroténoïdes (trichlorure d'antimoine), polyphénols et tannins (chlorure ferrique), flavonoïdes (réaction de la Cyanidine), stérols et terpènes (réaction de Lieberman), saponosides (présence de mousse persistance, indice de mousse) composés réducteurs (réactif de Fehling) et mucilages (éthanol 60²) (Evans, 2009).

\subsubsection{Chromatographie sur couche mince}

La méthode décrite par Dénou et al., (2016) a été utilisée.

\section{$>$ Dépôt des extraits}

Un volume d'environ $10 \mu \mathrm{L}$ de chaque extrait concentré a été déposé à l'aide de micropipettes sur une plaque de gel de silice ${ }_{60} \mathrm{GF}_{254}$ de $0,25 \mathrm{~mm}$ d'épaisseur.

\section{$>$ Migration des plaques}

Les plaques ont été séchées avant de les introduire dans une cuve de migration en verre contenant un mélange de Toluène - Acétate d'éthyle dans une proportion de $(95-5)$.

\section{$>$ Révélation des plaques}

Apres migration, les chromatogrammes obtenus ont été séchés et révélés par pulvérisation d'une solution d'anisaldéhyde suivie d'un chauffage.

\section{$>$ Calcul du rapport frontal (Rf)}

Apres révélation, le $\mathrm{Rf}$ des taches ont été calculé selon la formule suivante :

$$
\mathrm{Rf}=\frac{\mathrm{d}}{\mathrm{D}}
$$

Où $\mathrm{d}=$ distance parcourue par la tache $; \mathrm{D}=$ front du solvant 


\subsubsection{Caractérisation des constituants antiradicalaires}

Le chromatogramme des extraits migré dans le système de solvants Toluène - Acétate d'éthyle $(95$ - 5), a été pulvérisé avec une solution méthanolique de radical 2,2- diphenyl-1-picrylhydrazyl (DPPH) à 0,2\%. Les constituants antiradicalaires apparaissent sous forme de taches de couleur jaune sur fond violet [Jazy et al., 2018 ; Martson, 2011].

\section{Résultats et discussion}

\section{Enquête ethnobotanique.}

\subsection{Profil sociodémographique des personnes enquêtées}

Au total 75 personnes atteintes d'albinisme ont été enquêtées. Le sex ratio était de 0,70 en faveur du sexe féminin. Ce résultat est différent de celui de Gassama (2007) qui a obtenu un sex ratio de 1,1 en faveur du sexe masculin. Cette nouvelle prédominance féminine pourrait être due au fait qu'en 10 ans les femmes atteintes d'albinismes sont beaucoup plus alphabétisées et se tournent vers les associations pour une meilleure intégration.

Les personnes enquêtées sont des bambaras $(73,33 \%)$, des soninkés (14,7\%), des malinkés $(6,7 \%)$, des sénoufos $(4 \%)$ et des somonos $(1,3 \%)$. Ces résultats corroborent ceux de Gassama (2008) qui a trouvé aussi que les bambaras avec $(37,8 \%)$ étaient les plus représentées suivies des malinkés $(30 \%)$ et les soninkés $(16,4 \%)$. Ces pourcentages sont en accord avec la prédominance de bambaras, plus d'un tiers la population malienne [Cellule de Planification et de Statistiques, 2014].

\subsection{Recours à la phytothérapie}

Pour la protection et l'entretien de la peau, la majorité $(73,3 \%)$ des personnes enquêtées avaient eu recours aux plantes médicinales et 26,7\% n'avaient jamais eu recours aux plantes médicinales. Ce résultat confirme le grand recoures aux ressources de la médecine traditionnelle (MT) pour pallier aux problèmes de santé (World Health Organization, 2002).

\subsection{Espèces végétales inventoriées}

Au total 19 espèces végétales appartenant à 16 familles botaniques ont été recensées. Les espèces végétales les plus citées pour la protection cutanée des personnes atteintes d'albinisme sont Khaya senegalensis (Denv) A Juss (12 citations), Guiera senegalensis J.F.Gmel (10 citations), Bixa orellana L. (08 citations), et Vitellaria paradoxa C.F Gaertn. (05 citations) (Tableau I). L'utilisation de ces principales espèces végétales dans les soins de la peau et le traitement de certaines maladies de la peau avait été signalée dans la littérature : 
$>$ L'huile des graines de Khaya senegalensis est utilisée comme émollient cosmétique pour la peau et pour traiter diverses infections de la peau [Audu-Peter et al., 2006].

$>$ La racine de Guiera senegalensis est utilisée dans le traitement des inflammations et des blessures de la peau [Alshafei et al., 2016].

$>$ Les graines de Bixa orellana sont utilisées dans les soins de la peau, les infections et les brulures de la peau [Viuda-Martos et al., 2012].

- Le beurre de karité obtenu à partir des graines de Vitellaria paradoxa est utilisé comme émollient dans les crèmes et comme remèdes dans les brulures.

Des études antérieures ont démontrées que ces principales espèces végétales sont douées de propriétés pharmacologiques pouvant justifier leur utilisation dans les soins de la peau et le traitement de certaines maladies de la peau. En effet des études ont démontré les propriétés antioxydantes et antiinflammatoires de :

> Khaya senegalensis [Allah et al., 2018 ; Rabadeaux et al., 2017 ; Kolawole et al., 2012 ; Lompo et al., 2007] ;

$>$ Guiera senegalensis [Hamad et al., 2017 ; Jigam et al., 2011 ; Sombié et al., 2011] ;

> Bixa orellana |Djibersou et al., 2019 ; Yong et al., 2018 ; Radhika et al., 2017 ; Zarza-García et al., 2017 ; Ganju et Eisha, 2014 Yusuf et al., 2014 ; Yong et al., 2013] ;

$>$ Vitellaria paradoxa [Lin et al., 2018 ; Verma et al., 2012].

Les propriétés photoprotectrices de Bixa orellana ont été aussi démontrées par certains auteurs [Da Silva Caldas et al., 2020 ; Hussaana et al., 2019]. 
Tableau $\mathbf{N}^{\circ} \mathbf{I}$ : Espèces végétales utilisées pour la protection cutanée des personnes atteintes d'albinisme.

\begin{tabular}{|c|c|c|c|c|}
\hline $\mathbf{N}^{\circ}$ & Noms scientifiques (Familles) & Parties utilisées & Indications thérapeutiques & Nombre de citation \\
\hline 15 & Khaya senegalensis (Denv) A Juss (Meliaceae) & Fruits & Prévention de la peau & 12 \\
\hline 14 & Guiera senegalensis J.F.Gmel (Combretaceae) & Non définie & Entretien de la peau & 10 \\
\hline 7 & Bixa orellana $(\mathrm{L})($ Bixaceae) & Graines & Les brulures de la peau & 8 \\
\hline 18 & Vitellaria paradoxa C.F Gaertn. (Sapotaceae) & Graines & Entretien et protection de la peau & 5 \\
\hline 2 & Adansonia digatata $(\mathrm{L})$ (Malvaceae) & Non définie & Non définie & 4 \\
\hline 3 & Aloe vera (L) Burm, F. (Xanthorrhoeaceae) & Feuilles & Coup de soleil & 4 \\
\hline 4 & Ananas comosus (L.) Merr. (Bromeliaceae) & Fruits & Taches et point noir sur la peau & 2 \\
\hline 8 & Cocos nucifera $(\mathrm{L})$ (Arecaceae) & Fruits & Boutons et taches sur la peau & 2 \\
\hline 11 & Daucus carotta ( L) (Apiaceae) & Fruits & Non définie & 2 \\
\hline 13 & Ficus iteophylla Miq. (Moraceae) & Non définie & Non définie & 2 \\
\hline 17 & Sterculia setigera Delile (Malvaceae) & Feuilles & Entretien et protection de la peau & 2 \\
\hline 1 & Acacia nilotica (L) Delile (Leguminosae) & Graines & Boutons et taches sur la peau & 1 \\
\hline 5 & Annona muricata $(\mathrm{L})$ (Annonaceae) & Feuilles & Les parties lésées & 1 \\
\hline 6 & Balanites aegypticaca (L) Delile (Zygophyllaceae) & Graines & Boutons et taches sur la peau & 1 \\
\hline 9 & Curcuma longa $(\mathrm{L})$ (Zingiberaceae) & Fruits & Entretien de la peau & 1 \\
\hline 10 & Daniella oliveri Hutch \& Dalzel (Leguminosae) & Non définie & Entretien de la peau & 1 \\
\hline 12 & Elaeis guineensis Jacq (Arecaceae) & Non définie & Non définie & 1 \\
\hline 16 & Moringa oleifera Lam, (Moringaceae) & Feuilles & Embellissement de la peau & 1 \\
\hline 19 & Zea mays (L) (Poaceae) & Non définie & Non définie & 1 \\
\hline
\end{tabular}




\section{Analyse physicochimique des graines de Bixa orellana (L) (Bixaceae)} 2.1. Teneurs en eaux et en cendres

La teneur en eau était de 7,2\% inférieure à $10 \%$. Une teneur en eau élevée (généralement supérieure à $10 \%$ ) favorise la croissance des bactéries, des levures ou des champignons pendant le stockage du matériel végétal. Ces phénomènes peuvent altérer la qualité du principe actif lors de la conservation [Chanda, 2014]. Les teneurs en cendres totales et cendres insolubles dans l'acide chlorhydrique, ont été respectivement de $13,6 \%$ et 1,5\%. Ces données démontrent que la poudre des graines de Bixa orellana de notre étude est riche en éléments minéraux et est légèrement contaminée par le sable et la poussière [Chanda, 2014].

\subsection{Composition phytochimique}

L'analyse phytochimique la poudre des graines de Bixa orellana a révélé la présence des caroténoïdes, des oses et holosides, des mucilages par les réactions colorées en tube et des composés terpéniques qui sont apparus en violet après révélation avec l'anisaldéhyde (Figure $\mathrm{N}^{\circ} 1$ ).

Par contre les alcaloïdes, anthracénosides, tanins, flavonoïdes et saponosides étaient absents. Ces résultats sont différents de ceux reportés dans la littérature. En effet des études ont démontré la présence des anthracénosides, flavonoïdes, saponosides, tanins dans les graines de Bixa orellana, [Abayomi et al., 2014 ; Ganju et Eisha, 2014 ; Oboh et al., 2011 ; Tamil et al., 2011].

La présence des caroténoïdes et des mucilages dans la poudre des graines de Bixa orellana, peuvent justifier leur utilisation dans la protection et l'entretien de la peau des personnes atteintes d'albinisme. Les caroténoïdes sont reconnus pour leur effet photo-protecteur et les mucilages pour leur effet hydratant [Bruneton, 2016]. Il est important de noter que les caroténoïdes sont préconisés en photodermatoses puisqu'ils interfèrent avec les processus de photo-oxydation [Krief, 2003]

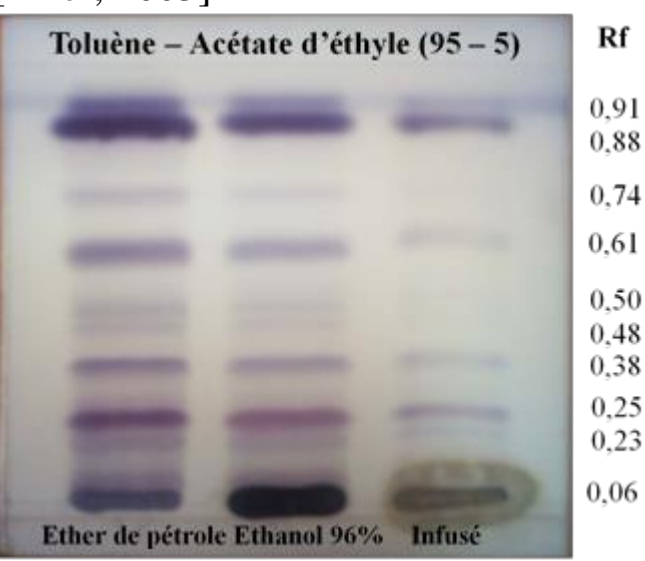

Figure 1 : Chromatogramme des extraits graines de Bixa orellana, migré dans le système Toluène - Acétate d'éthyle $(95$ - 5) puis révélé par une solution d'anisaldéhyde et chaffage. 


\subsection{Constituants antiradicalaires}

L'apparition d'une tâche jaunâtre sur fond violet au Rf 0,25 pour les extraits éther de pétrole et éthanol $96 \%$ pourrait être due à la présence de constituant antiradicalaire (Figure $\mathrm{N}^{\circ} 2$ ). De nombreux travaux ont démontré l'activité antiradicalaire des extraits des graines de Bixa orellana [Abayomi et al., 2014 ; Viuda-Martos et al., 2012]. Cette activité antiradicalaire pourrait être lié à la présence de caroténoïdes qui sont connus pour leur propriété antioxydante [Bruneton, 2016 ; Stah et Sies, 2003].

Les rayons ultra-violet (UV) induisent la production des radicaux libres qui entrainent une peroxydation lipidique, une altération des protéines, une inflammation et une immunosuppression [Bosch et al., 2015].

Les propriétés antiradicalaires des extraits des graines de Bixa orellana pourraient justifier l'utilisation de la plante dans la protection cutanée.

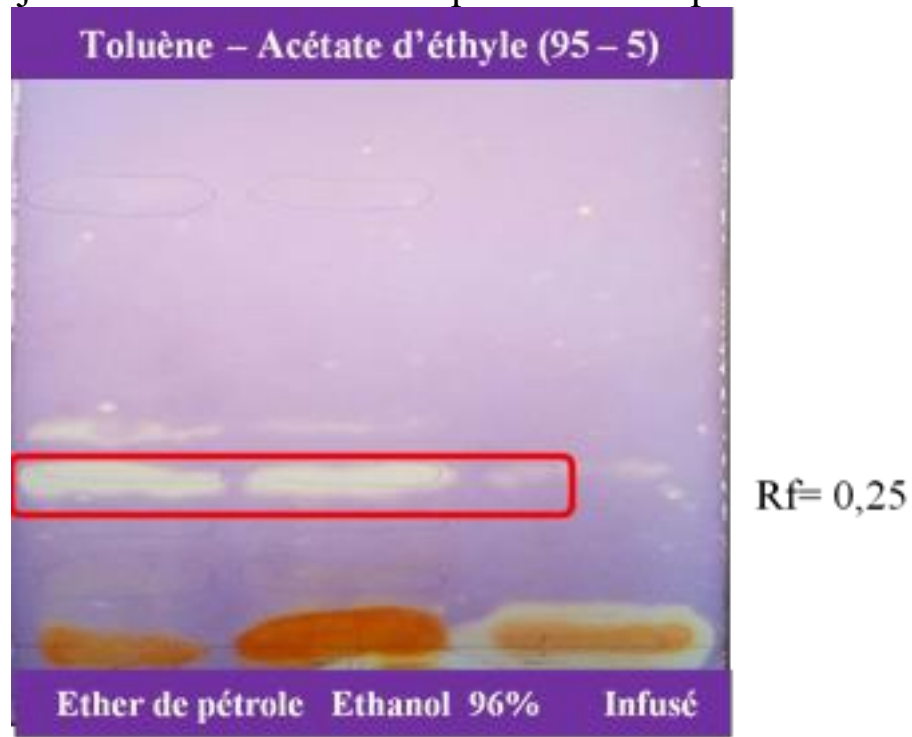

Figure 2 : Chromatogramme des extraits graines de Bixa orellana, migré dans le système Toluène - Acétate d'éthyle $(95-5)$ puis révélé par le DPPH.

\section{Conclusion et perspectives}

Les personnes atteintes d'albinisme oculo-cutané (AOC) au Mali ont recours à l'utilisation des plantes pour la protection et l'entretien cutanés. Parmi ces plantes, Bixa orellana, est riche en caroténoïdes, mucilages, stérols, triterpènes et constituants antiradicalaires (caroténoïdes). Ces constituants peuvent contribuer à la photo-protection, l'hydratation et la protection cutanée des personnes atteintes d'albinisme.

En perspective, nous envisageons incorporer les extraits des graines de Bixa orellana, qui sont riches en caroténoïdes, dans du beurre de karité pour la mise au point de produits destinés à la protection de la peau. 


\section{References:}

1. Abayomi, M., Adebayo, A. S., Bennett, D., Porter, R., \& ShellyCampbell, J. (2014). In vitro antioxidant activity of Bixa orellana (Annatto) seed extract. Journal of Applied Pharmaceutical. Science, 4 (2), $101-106$.

2. Abayomi, M., Adebayo, A. S., Bennett, D., Porter, R., Campbell, J. S., \& Dawkin, G. (2014). Phytochemical testing and in vitro antibacterial activity of Bixa orellana (Annatto) seed extract. British Journal of Pharmaceutical Research, 4 (11), 1387-1399.

3. Al Shafei, N. K., Elshafie, A. E., \& Nour, A. (2016). Antitoxic, antifungal and phytochemical analysis of medicinal compounds of Guiera senegalensis leaves in Sudan. Journal of Plant Biochemistry \& Physiology, 4 (2), 1-4.

4. Allah, M. O. W., Alrasheid, A. A., \& Elamin, A. S. (2018). Phytochemical screening, chemical composition and antioxidant activity of leaves and bark extracts from Khaya senegalensis. Advances in Biochemistry, 6(4), 32 - 38.

5. Audu-Peter, J. D., Olorunfemi, P. O., \& Njoku, N. (2006). Antimicrobial and pharmaceutical properties of Khaya senegalensis seed oil. Journal of Pharmacy and Bioresources, 3(1), 19-24.

6. Bruneton, J. (2016). Pharmacognosy, phytochemistry, medicinal plants. Lavoisier publishing. $5^{\text {ème }}$ edition. 1487 p.

7. Cellule de Planification et de Statistiques (CPS/SSDSPF), 2014. Enquête Démographique et de Santé (EDSM-V) 2012 - 2013, Mali. P577

8. Chanda, S., 2014. Importance of pharmacognostic study of medicinal plants: An overview. Journal of Pharmacognosy and Phytochemistry 2 (5), $69-73$.

9. Da Silva Caldas, D. C., Diniz, R. C., do Carmo, L. H. A., Batista, M. C. A., \& Vilanova, C. M. (2020). Influence of the incorporation of annatto oil Bixa orellana $\mathrm{L}$. in the sun protection factor by visible spectroscopy of photoprotective formulations. International Journal of Development Research, 10(01), 33285-33292.

10. Evans, W. C. (2009). Trease and Evans Pharmacognosy 16th ed. Saunders.

11. Ganju, K., \& Ganju, E. (2014). Phytochemical Analysis of Seeds of Bixa orellana Linn. Journal of Medical and Pharmaceutical Innovation, 1(3), 21-24.

12. Gassama, M. (2008). Aspects épidemio-cliniques, et prise en charge dermatologique des albinos dans le service de dermato- vénéreologie du CNAM (ex Institut Marchoux). Bamako (Mali) (Doctoral dissertation, Thèse Pour obtenir le grade de Docteur d'état en 
Médecine, Faculté de Médecine, de Pharmacie Et d'Odontostomatologie 2007-2008, 87p).

13. Grønskov, K., Ek, J., \& Brondum-Nielsen, K. (2007). Oculocutaneous albinism. Orphanet journal of rare diseases, 2(1), 43.

14. Hamad, M. S., Hassan, E., Ahmed, S., Fadul, E., \& Ahmed, R. H. (2017). A review on the taxonomy, ethnobotany, phytochemistry and pharmacology of Guriea senegalensis J.F. Gmel. (Combretaceae). Journal of Medicinal and Aromatic Plants (Los Angeles), 6 (296), 2167-0412.

15. https://www.un.org/fr/events/albinismday/background.shtml consulté le 04/09/2019 à 12 h 43 mn.

16. http://www.theplantlist.org/ consulté le 05/11/2019 à 10 h 30 mn

17. Jazy, M. A., Haidara M, \& Sanogo, R. (2018). Chromatographie sur couche mince et activité antiradicalaire d'extraits de Pupalia Lappacea (L.) Juss. Amaranthaceae. European Scientific Journal, ESJ, 14(3), 140.

18. Jigam, A. A., Akanya, H. O., Dauda, B. E., \& Ogbadoyi, E. O. (2011). Antiplasmodial, analgesic and anti-inflammatory effects of crude Guiera senegalensis J.F. Gmel. (Combretaceae) leaf extracts in mice infected with Plasmodium berghei. Journal of Pharmacognosy and Phytotherapy, 3(10), 150-4.

19. Kolawole, O. T., Kolawole, S. O., Ayankunle, A. A., \& Olaniran, O. I. (2012). Anti-hyperglycemic effect of Khaya senegalensis stem bark aqueous extract in Wistar rats. European Journal of Medicinal Plants, 66-73.

20. Krief Sabrina,(2003). Métabolites secondaires des plantes et comportement animal : surveillance sanitaire et observations de l'alimentation des chimpanzés (Pan troglodytes schweinfurthii) en Ouganda. Activités biologiques et étude chimique de plantes consommées. Sciences du Vivant [q-bio]. Museum national d'histoire naturelle - MNHN PARIS. Français. 〈tel-00006170〉

21. Lin, T. K., Zhong, L., \& Santiago, J. L. (2018). Anti-inflammatory and skin barrier repair effects of topical application of some plant oils. International journal of molecular sciences, 19(70), 1-21.

22. N'Guessan Bra Y.F, Sanogo R, Coulibaly K \& Diénéba, K. B. (2015). Minerals salt composition and secondary metabolites of Euphorbia hirta Linn., an antihyperglycemic plant. Pharmacognosy research, $7(1), 7-13$.

23. Oboh, G., Akomolafe, T. L., Adefegha, S. A., \& Adetuyi, A. O. (2011). Inhibition of cyclophosphamide-induced oxidative stress in rat brain by polar and non-polar extracts of Annatto (Bixa orellana) seeds. Experimental and toxicologic pathology, 63(3), 257-262. 
24. Rabadeaux, C., Vallette, L., Sirdaarta, J., Davis, C., \& Cock, I. E. (2017). An examination of the antimicrobial and anticancer properties of Khaya senegalensis (Desr.) A. Juss. bark extracts. Pharmacognosy Journal, 9(4), 504-518

25. Rooryck, C., Morice, F., Mortemousque, B., Lacombe, D., Taïeb, A., \& Arveiler, B. (2007). Albinisme oculo-cutané. Annales de dermatologie et de vénéréologie, 134, 55-64. Elsevier.

26. Sharma, P., Kharkwal, A. C., Kharkwal, H., Abdin, M. Z., \& Varma, A. (2014). A review on pharmacological properties of Aloe vera. International Journal of Pharmaceutical Science Review and Research, 29(2), 31-37.

27. Sombié, P. A. E. D., Hilou, A., Mounier, C., Coulibaly, A. Y., Kiendrebeogo, M., Millogo, J. F., \& Nacoulma, O. G. (2011). Antioxidant and anti-inflammatory activities from galls of Guiera senegalensis JF Gmel. (Combretaceae). Research Journal of Medicinal Plant, 5(4), 448-461.

28. Tamil Selvi, A., Dinesh, M. G., Satyan, R. S., Chandrasekaran, B., \& Rose, C. (2011). Leaf and Seed extracts of Bixa orellana L. exert antimicrobial activity against bacterial pathogens. Journal of Applied Pharmaceutical Science, 1(9), 116-120.

29. Verma, N., Chakrabarti, R., Das, R. H., \& Gautam, H. K. (2012). AntiInflammatory effects of shea butter through inhibition of iNOS, COX2, and cytokines via the $\mathrm{Nf}-\mathrm{Kb}$ pathway in LPS-activated $\mathrm{J} 774$ macrophage cells. Journal of Complementary and Integrative Medicine, 9(1), 1-11.

30. Viuda-Martos, M., Ciro-Gómez, G. L., Ruiz-Navajas, Y., ZapataMontoya, J. E., Sendra, E., Pérez-Álvarez, J. A., \& Fernández-López, J. (2012). In vitro antioxidant and antibacterial activities of extracts from annatto (Bixa orellana L.) Leaves and Seeds. Journal of Food Safety, 32(4), 399-406.

31. World Health Organization, (2002). Stratégie de l'OMS pour la Médecine Traditionnelle pour 2002-2005 ( ${ }^{\circ}$ WHO/EDM/TMR/2002.1). Genève : Organisation mondiale de la santé.

32. Yong, Y. K., Somchit, M. N., \& Ahmad, Z. (2018). Anti-inflammatory properties of Bixa orellana leaves extract are associated with suppression of bradykinin-induced endothelial hyperpermeability. Pharmacognosy Magazine, 14(57), 352 - 7

33. Yong, Y. K., Zakaria, Z. A., Kadir, A. A., Somchit, M. N., Lian, G. E. C., \& Ahmad, Z. (2013). Chemical constituents and antihistamine activity of Bixa orellana leaf extract. Complementary and alternative medicine, 13(1), 1-7. 
34. Zarza-García, A. L., Sauri-Duch, E., Raddatz-Mota, D., Cuevas-Glory, L. F., Pinzón-López, L. L., Rivera-Cabrera, F., \& Mendoza-Espinoza, J. (2017). Pharmacological, phytochemical and morphological study of three Mayan accessions of Bixa orellana L. leaves. Emirates Journal of Food and Agriculture, 163-169. 